\title{
Transformações mineralógicas e cristaloquímicas decorrentes dos ensaios termais em argilas cauliníticas ferruginosas
}

\section{(Mineralogical and crystalochemical transformations originated from thermal essays on ferruginous kaolinitic clays)}

\author{
M. M. Ferreira' ${ }^{1}$ A. F. D. C. Varajão ${ }^{1}$, A. M. Morales-Carrera ${ }^{1}$, M. G. Peralta-Sánchez ${ }^{1}$, G. M. da Costa ${ }^{2}$ \\ ${ }^{1}$ Departamento de Geologia, Escola de Minas \\ ${ }^{2}$ Departamento de Química, ICEB \\ Universidade Federal de Ouro Preto, Campus Universitário, Morro do Cruzeiro s/n, Ouro Preto, MG 35400-000 \\ angelica@degeo.ufop.br
}

\begin{abstract}
Resumo
Transformações mineralógicas e cristaloquímicas foram investigadas em amostras de argilas cauliníticas ferruginosas aquecidas a temperaturas de 800,1000 e $120{ }^{\circ} \mathrm{C}$. As análises de difração de raios X e térmica diferencial e gravimétrica mostraram ser a caulinita o argilomineral predominante nas amostras naturais. Os resultados da espectroscopia Mössbauer mostraram que o alto conteúdo em $\mathrm{Fe}(22,5 \% \mathrm{em}$ peso), determinado por fluorescência de raios X, é relacionado à presença de goethita $(18 \%$ em peso) e hematita (16\% em peso). Entretanto um resíduo deste elemento $\left(\mathrm{Fe}^{3+}\right.$ e $\left.\mathrm{Fe}^{2+}\right)$ foi constatado após a extração do ferro usando solução de ditionito-citrato-bicarbonato, e possivelmente se deve à presença de ferro na estrutura da caulinita. O processo de sinterização mostra uma desestruturação da caulinita, assim como a transformação da goethita em hematita, cujo tamanho dos cristais cresce com o incremento da temperatura. $\mathrm{O}$ tamanho do cristal de hematita a $1200^{\circ} \mathrm{C}$ é 5 vezes maior do que na amostra natural. A mulita formada a $1000{ }^{\circ} \mathrm{C}$ é constituída da solução sólida de $\mathrm{Fe}_{2} \mathrm{O}_{3}$, com $\mathrm{Al}_{2} \mathrm{O}_{3}$ o que resulta em produto resistente e de maior estabilidade térmica.
\end{abstract}

Palavras-chave: caulim ferruginoso, mulita, solução sólida de $\mathrm{Fe}_{2} \mathrm{O}_{3}$ e $\mathrm{Al}_{2} \mathrm{O}_{3}$, espectroscopia Mössbauer.

Abstract

\begin{abstract}
Mineralogical and crystallochemical transformations of representative ferruginous kaolinitic clay samples were investigated in specimens burned at 800,1000 and $1200^{\circ} \mathrm{C}$. X-ray diffraction and differential thermal and thermogravimetric analyses showed that kaolinite was the predominant mineral in the raw samples. The Mössbauer spectroscopy results showed that the high iron content (22.5 wt.\%), as determined by X-ray fluorescence, is related to the presence of goethite (18wt.\%) and hematite (16 wt.\%). However, after $\mathrm{Fe}$ was extracted using a dithionite-citrate system buffered with sodium bicarbonate, a residue of this element ( $\mathrm{Fe}^{3+}$ and $\left.\mathrm{Fe}^{2+}\right)$ was found in the structure of the kaolinite. The sintering process showed the destruction of kaolinite, as well as the transformation of the goethite into hematite, the crystals growing as the temperature increases. The hematite crystal size at $1200{ }^{\circ} \mathrm{C}$ is five times larger than in the raw sample. The mullite formation at $100{ }^{\circ} \mathrm{C}$ is comprised of a solid solution of $\mathrm{Fe}_{2} \mathrm{O}_{3}$ and $\mathrm{Al}_{2} \mathrm{O}_{3}$, which results in a resistant product with a higher thermal stability.
\end{abstract}

Keywords: ferruginous kaolin, mullite, solid solutions of $\mathrm{Fe}_{2} \mathrm{O}_{3}$ and $\mathrm{Al}_{2} \mathrm{O}_{3}$, Mössbauer spectroscopy.

\section{INTRODUÇÃO}

Estudos realizados nos corpos argilosos cauliníticos do Quadrilátero Ferrífero visando à análise da viabilidade de sua aplicação na indústria cerâmica [1-3] mostraram que apenas as argilas ferruginosas usadas no seu estado natural cumpriram as especificações técnicas para cerâmica com a obtenção de corpos cerâmicos sem fraturas e com altos valores de resistência à compressão. Estes estudos foram apoiados nos trabalhos de caracterização faciológica dos depósitos de caulim [4] que resultaram na definição morfológica, mineralógica e química das fácies de algumas ocorrências, dados estes imprescindíveis para estudos aplicativos. Neste sentido o presente trabalho teve como objetivo definir os processos atuantes durante os ensaios termais onde as transformações mineralógicas e cristaloquímicas foram caracterizadas. A matéria-prima utilizada foi proveniente do depósito de caulim Padre Domingos [4].

\section{MATERIAIS E MÉTODOS}

Foram utilizadas amostras representativas da fácies Diamictito Vermelho (DV) do depósito de caulim Padre Domingos [4], localizado no Quadrilátero Ferrífero, às margens da rodovia BR-040 (Fig. 1). A amostragem foi feita com a coleta de $10 \mathrm{~kg}$ de amostra em cinco pontos diferentes 
da respectiva fácies, distribuídos da base ao topo. Uma única amostra composta DV foi obtida após os processos de secagem, destorroamento, homogeneização e quarteamento. Os dados de análise granulométrica por peneiramento a úmido e granulômetro a laser, assim como os limites de liquidez e o índice de plasticidade [1-3] foram utilizados como suporte neste trabalho.
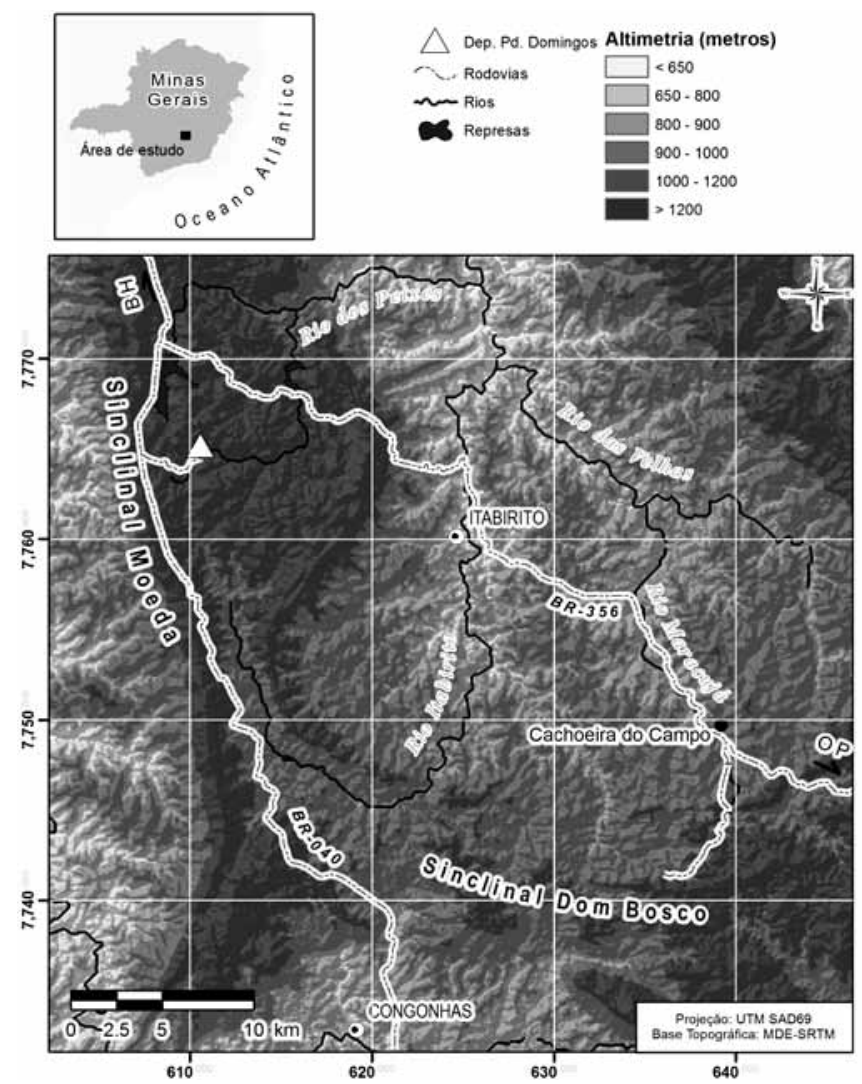

Figura 1: Localização do depósito de caulim de Padre Domingos, Quadrilátero Ferrífero, Minas Gerais.

[Figure 1: Localization of kaolin deposit of Padre Domingos in Quadrilátero Ferrifero, Minas Gerais, Brazil.]

Para a preparação dos corpos de prova a amostra composta foi peneirada a \#35 mesh $(0,425 \mathrm{~mm})$ e corpos de prova em forma de pastilhas com aproximadamente 0,3 $\mathrm{cm}$ de altura e $0,7 \mathrm{~cm}$ de diâmetro foram obtidos a partir de $0,8 \mathrm{~g}$ de amostra com $8,5 \%$ de umidade [5] em prensa hidráulica Alfred Jamsler. As pastilhas foram submetidas a ensaios térmicos em um forno tubular com resistência de super-kanthal controlado por computador, com aquecimento máximo de $1700{ }^{\circ} \mathrm{C}$. As pastilhas foram aquecidas a 800 , 1000 e $1200{ }^{\circ} \mathrm{C}$ por $5 \mathrm{~h}$.

A caracterização mineralógica foi obtida em um difratômetro de raios X Shimadzu XRD 6000 com tubo de radiação $\mathrm{k \alpha Fe} e$ monocromador de grafite. Para a aquisição do difratograma da fração total da amostra natural pulverizada (passante \#35 mesh) e dos corpos de prova calcinados em diferentes temperaturas, analisaramse lâminas desorientadas a $1,2^{\circ} / \mathrm{min} \operatorname{com} 2 \theta$ de 2 a $70^{\circ}$. As lâminas foram obtidas a partir da deposição e distribuição homogênea dos pós das amostras na cavidade de uma lâmina de vidro, seguida pelo nivelamento da sua superfície, sem pressioná-la para evitar possível orientação. Adicionalmente, foi feita decomposição dos difratogramas em $2 \theta$ de 40 a $44^{\circ}$ em curvas Gaussianas por meio do Programa Jade +, versão 9 (MDI) para a determinação das fases coincidentes com o pico $(d=2,70 \AA)$ da hematita.

Análise Térmica Diferencial (ATD) e Análise Térmica Gravimétrica (ATG) foram feitas utilizando equipamento TA Inst. 2960 STD V3.0F, com um porta amostra de platina e de alumina (padrão) onde a amostra foi introduzida sob atmosfera de nitrogênio com taxa de aquecimento $20^{\circ} \mathrm{C} / \mathrm{min}$ até $1100^{\circ} \mathrm{C}$.

Espectros Mössbauer para obtenção de dados cristaloquímicos e cristalográficos das fases ferruginosas presentes na amostra bruta, na amostra após tratamento de deferrificação e na amostra aquecida a 800,1000 e $1200{ }^{\circ} \mathrm{C}$, foram obtidos em um espectrômetro à temperatura ambiente em modo de aceleração constante e acumulação de dados em analisador multicanal com 512 canais. A preparação dos absorvedores se deu com 10 a $20 \mathrm{mg}$ de amostra e os espectros obtidos apresentaram sextetos e/ou dubleto simétricos, a partir dos quais foram determinadas as áreas relativas de cada componente.

A deferrificação da amostra foi feita adotando-se a metodologia adaptada [6] com $20 \mathrm{~g}$ de amostra tratada com solução de citrato-bicarbonato e ditionito (DCB). Amostras da argila natural pulverizada e também da argila deferrificada por DCB foram submetidas à análise química dos elementos maiores e menores, em um aparelho de fluorescência de raios X Philips PW2404 MagiX com amostrador automático PW2504 e tubo de Rh a 2,4 kW.

\section{RESULTADOS E DISCUSSÃO}

A mineralogia da amostra DV é constituída predominantemente por caulinita $(\mathrm{K})$ e secundariamente por hematita (H), goethita (Go) e muscovita (MS) (Fig. 2A). Após os tratamentos térmicos identificou-se a presença da hematita $(\mathrm{H})$ nas diferentes temperaturas de sinterização. A muscovita (MS) persiste apenas até $800{ }^{\circ} \mathrm{C}$. A $1000{ }^{\circ} \mathrm{C}$ tem-se a mulita (ML), produto originado da queima/fusão de compostos silico-aluminosos. A cristobalita fica bem evidenciada quando a amostra é aquecida a $1200^{\circ} \mathrm{C}$.

$\mathrm{O}$ aumento da temperatura de queima também gera aumento do tamanho dos grãos da hematita [7-10]. A partir da decomposição dos difratogramas (Fig. 2B) e baseado na equação de Scherrer [11], que determina o diâmetro médio do cristal, foi possível estimar o tamanho médio do cristal de hematita (Tabela I). Para isso, foi considerado o pico que representa o plano $104(d=2,70 \AA)$ dos cristais da hematita, por ser o pico que apresentou menor interferência dos outros minerais. Assim, o grão da hematita possui um valor de $160 \AA$ que cresce para $220 \AA$ quando queimada a $800^{\circ} \mathrm{C}$, para $290 \AA$ a $1000^{\circ} \mathrm{C}$ e, é maior do que $1000 \AA$ quando atinge $1200{ }^{\circ} \mathrm{C}$. O pequeno desvio de $0,326^{\circ} 2 \theta$ do pico da hematita para ângulos maiores desde a amostra 
(a)

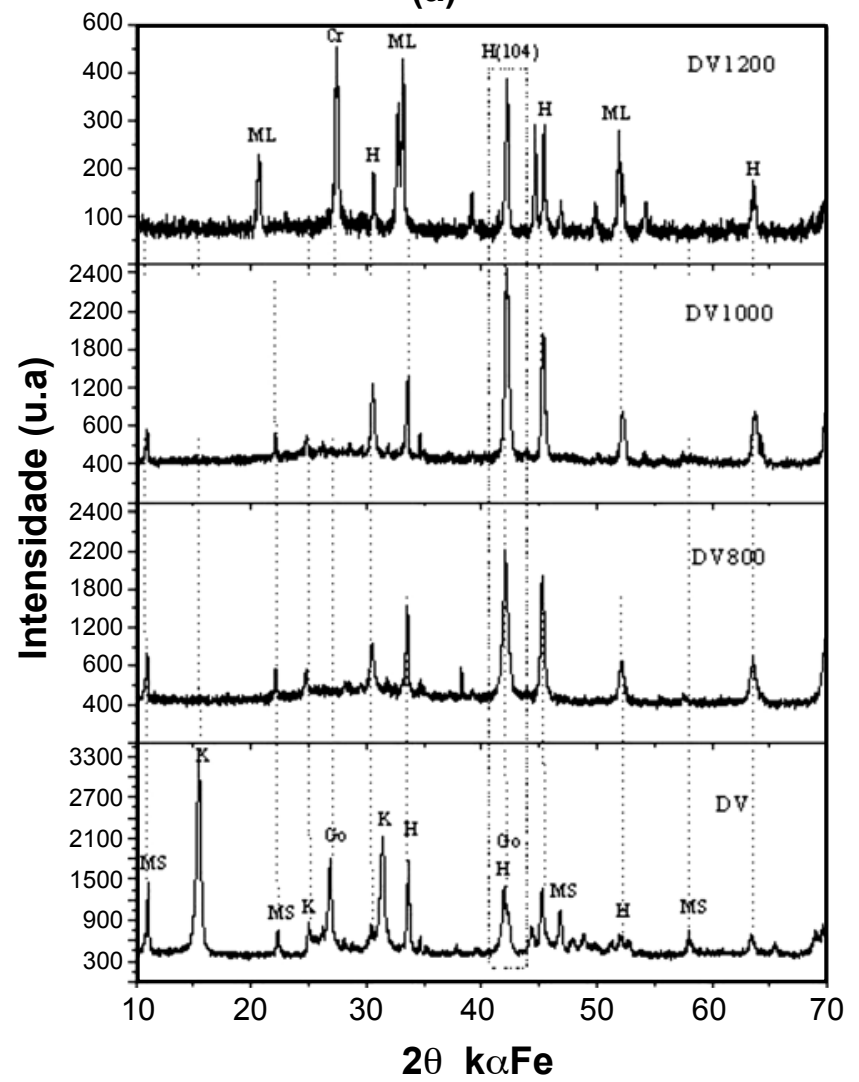

(b)

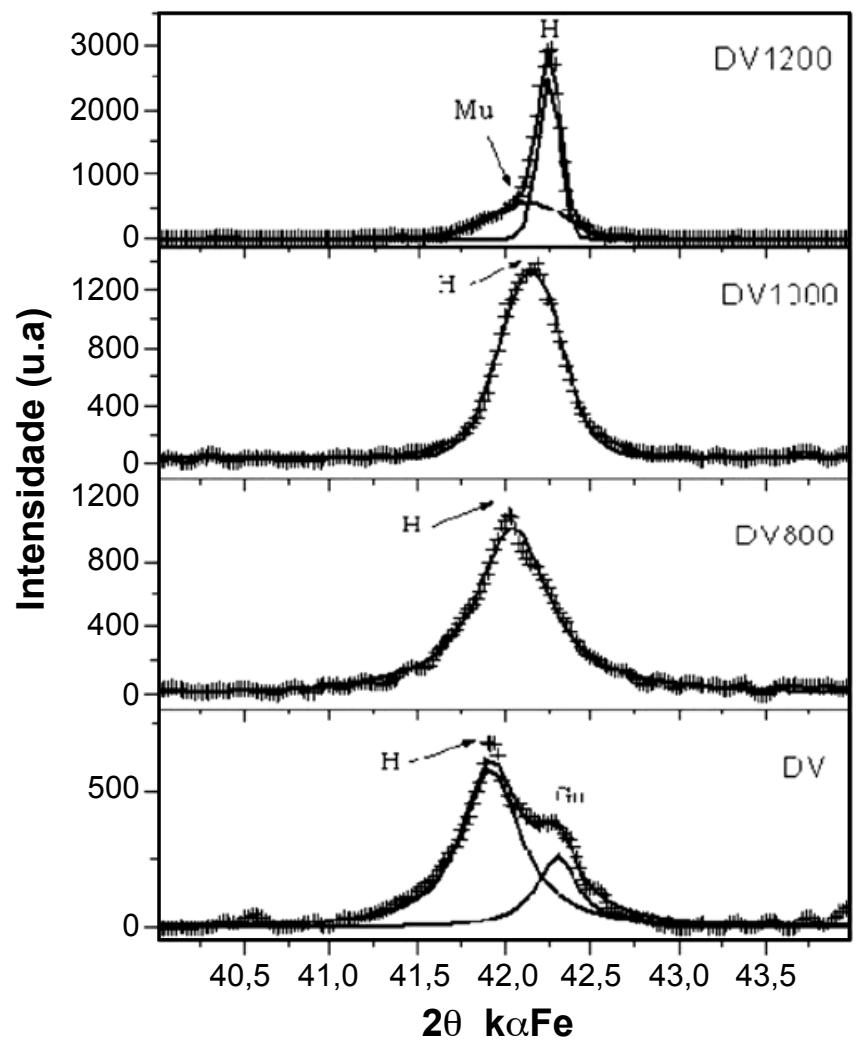

Figura 2: A) Difratogramas de raios $\mathrm{X}$ (radiação $\mathrm{k} \alpha$ de $\mathrm{Fe}$ ) da amostra $\mathrm{DV}$ natural e aquecida a 800,1000 e $1200{ }^{\circ} \mathrm{C} . \mathrm{K}=\mathrm{caulinita}, \mathrm{H}=$ hematita, $\mathrm{Q}=$ quartzo, $\mathrm{Fd}=$ feldspato, $\mathrm{MS}=$ muscovita, $\mathrm{Go}=$ goethita, $\mathrm{ML}=$ mulita (A) e detalhe da variação do pico (104) da hematita de acordo com o aumento da temperatura (B); B) Decomposição dos difratogramas de raios X no intervalo de $2 \theta 41-44^{\circ}$.

[Figure 2: A) X-ray diffraction patterns (Feka radiation) of the natural sample DV and burned at 800,1000 and $1200{ }^{\circ} \mathrm{C} . \mathrm{K}=\mathrm{kaolinite}$, $H=$ hematite, $Q=$ quartz, $M S=$ muscovite, Go = goethite, $M L=$ mullite; B) Decomposition of X-ray diffraction patterns (Fek radiation) in the $41-44^{\circ} 2 \theta$ range.]

natural DV até DV1200, que corresponde a uma variação de 2,706 $\AA$ (DV) para 2,688 $\AA$ (DV1200), pode ser devido a desvios instrumentais como preparação das amostras e/ou porta amostra [12]. Desvios instrumentais podem chegar a $0,2^{\circ}$ em $2 \theta$ e, no caso deste estudo, o desvio de $\sim 0,33^{\circ} 2 \theta$ de DV para DV1200 pode ser devido a uma combinação de desvios nas duas medidas (DV800 e DV1000).

A análise térmica diferencial (Fig. 3) corrobora o difratograma de raios $\mathrm{X}$, apresentando um pico endotérmico a $490{ }^{\circ} \mathrm{C}$, que correspondente à desidroxilação da caulinita [13]. Este baixo valor pode estar associado à caulinita com defeitos estruturais [14]. Nota-se também a presença de um pico endotérmico proeminente a $307^{\circ} \mathrm{C}$, podendo caracterizar a presença da goethita, que se transforma em hematita acima desta temperatura.

A composição química da amostra natural (Tabela I) mostra a presença relevante de óxidos $\mathrm{SiO}_{2}$ e $\mathrm{Al}_{2} \mathrm{O}_{3}$ seguidos pelo $\mathrm{Fe}_{2} \mathrm{O}_{3}$, sendo que este pode ser o responsável pela coloração avermelhada do material. Depois da retirada do Fe livre da amostra por tratamento DCB, há uma redução do teor de $\mathrm{Fe}$, passando de $32,25 \%$ para $0,87 \%$, atingindo a eliminação de $97 \%$ do Fe inicial, possivelmente presente na forma de óxidos e hidróxidos. A presença remanescente deste elemento pode estar relacionada ao $\mathrm{Fe}^{3+}$ presente

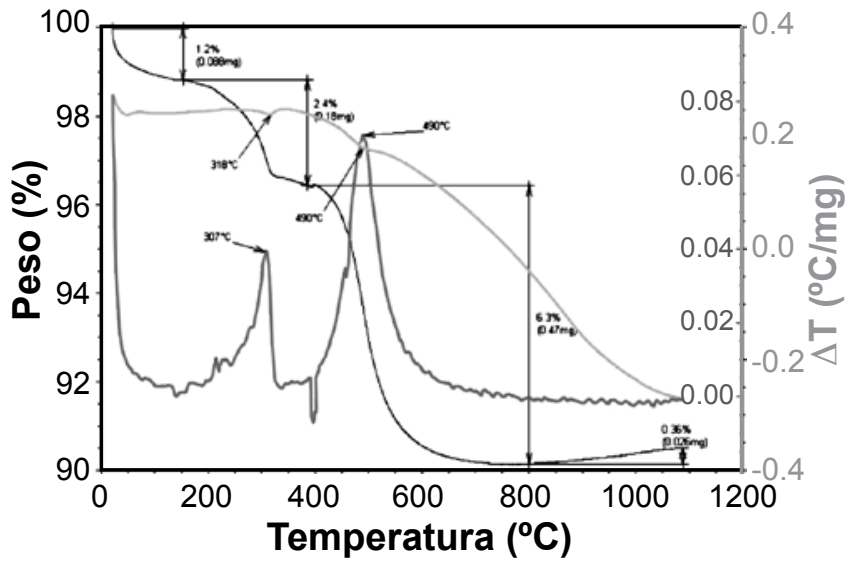

Figura 3: Curvas de análise térmica diferencial e gravimétrica da amostra DV. Notar o pico endotérmico da goethita a $307^{\circ} \mathrm{C}$ e da caulinita a $490^{\circ} \mathrm{C}$.

[Figure 3: Differential thermal (DTA) and thermogravimetric analysis (TGA) patterns of DV sample. Note the endothermic peak of goethite at $307^{\circ} \mathrm{C}$ and kaolinite at $490{ }^{\circ} \mathrm{C}$.] 
Tabela I - Composição química antes (DV) e após a extração do Fe (DVT). PPC = perda por calcinação. \% = porcentagem em peso.

[Table I - Chemical composition before (DV) and after extractable Fe (DVT). PPC = loss on ignition. \% = wt.\%.]

\begin{tabular}{ccccccccccccc}
\hline \multirow{2}{*}{ Amostra } & $\begin{array}{c}\mathrm{SiO}_{2} \\
(\%)\end{array}$ & $\begin{array}{c}\mathrm{Al}_{2} \mathrm{O}_{3} \\
(\%)\end{array}$ & $\begin{array}{c}\mathrm{Fe}_{2} \mathrm{O}_{3} \\
(\%)\end{array}$ & $\begin{array}{c}\mathrm{TiO}_{2} \\
(\%)\end{array}$ & $\begin{array}{c}\mathrm{MnO} \\
(\%)\end{array}$ & $\begin{array}{c}\mathrm{MgO} \\
(\%)\end{array}$ & $\begin{array}{c}\mathrm{CaO} \\
(\%)\end{array}$ & $\begin{array}{c}\mathrm{Na}_{2} \mathrm{O} \\
(\%)\end{array}$ & $\begin{array}{c}\mathrm{K}_{2} \mathrm{O} \\
(\%)\end{array}$ & $\begin{array}{c}\mathrm{P}_{2} \mathrm{O}_{5} \\
(\%)\end{array}$ & $\begin{array}{c}\mathrm{PPC} \\
(\%)\end{array}$ & $\begin{array}{c}\text { Total } \\
(\%)\end{array}$ \\
\hline $\mathrm{DV}$ & 32,16 & 22,97 & 32,25 & 1,11 & 0,01 & 0,04 & 0,02 & 0,072 & 0,98 & 0,238 & 10,11 & 99,96 \\
$\mathrm{DVT}$ & 52,48 & 32,69 & 0,87 & 1,39 & 0,00 & 0,11 & 0,03 & 0,302 & 1,69 & 0,026 & 0,00 & 89,59 \\
$\mathrm{DVT} / \mathrm{DV}$ & 1,63 & 1,42 & 0,03 & 1,25 & 0,00 & 2,75 & 1,50 & 4,29 & 1,72 & 0,11 & 0,00 & 0,90 \\
\hline
\end{tabular}

em sítios octaédricos das caulinitas como substituinte do $\mathrm{Al}^{3+}[13,14]$. A relação $\mathrm{SiO}_{2} / \mathrm{Al}_{2} \mathrm{O}_{3}$ de 1,40 na amostra DV natural aumenta para 1,61 após deferrificação, como resultado do aumento proporcional dos compostos químicos após a eliminação do $\mathrm{Fe}_{2} \mathrm{O}_{3}$, considerando que parte do $\mathrm{Al}$ pode estar na estrutura dos óxidos e hidróxidos de ferro eliminados (Tabela I). Alem disso, a utilização de reagentes no tratamento por DCB acarreta a adição de elementos, principalmente o sódio que apresenta um acréscimo de mais de quatro vezes na amostra DVT (Tabela I).

Os espectros Mössbauer (Fig. 4) anteriormente ao tratamento de deferrificação revelam a presença de um dubleto característico da presença de $\mathrm{Fe}^{3+}$; o espectro é também composto por um sexteto relativamente estreito devido à existência de hematita, corroborando os resultados do difratograma de raios $\mathrm{X}$, e um sexteto largo provavelmente devido à presença de goethita de baixa cristalinidade e/ou alta substituição isomórfica. A presença deste sexteto estreito relativo à presença de hematita persiste até a temperatura de $1200{ }^{\circ} \mathrm{C}$. A $1000{ }^{\circ} \mathrm{C}$ associa-se a este sexteto um dubleto característico da presença de $\mathrm{Fe}^{3+}$ que torna bem evidente a $1200{ }^{\circ} \mathrm{C}$, sendo correlacionado a soluções sólidas de óxido de ferro na mulita. Com o aquecimento a partir de 1000 ${ }^{\circ} \mathrm{C}$ o $\mathrm{Fe}_{2} \mathrm{O}_{3}$ com $\mathrm{Al}_{2} \mathrm{O}_{3}$ forma soluções sólidas de óxido de ferro na mulita, resultando produtos resistentes e com maior estabilidade térmica [15].

Nos espectros da amostra natural após o tratamento com DCB observa-se que o dubleto de $\mathrm{Fe}^{3+}$ permanece, indicando

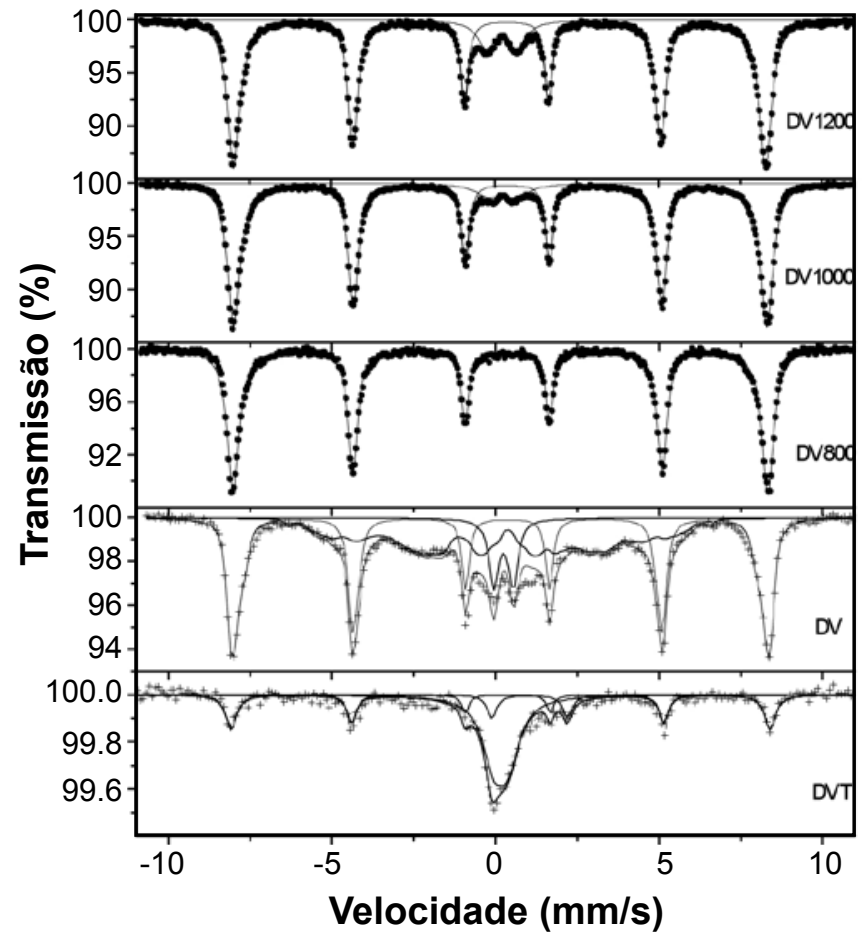

Figura 4: Espectros Mössbauer à temperatura ambiente da amostra natural antes (DV) e após (DVT) ser submetida ao tratamento DCB e das amostras aquecidas a 800,1000 e $1200{ }^{\circ} \mathrm{C}$.

[Figure 4: Mössbauer spectra at room temperature of the natural DV sample, before and after extractable Fe and burned at 800, 1000 and $1200^{\circ} \mathrm{C}$.]

Tabela II - Parâmetros hiperfinos obtidos dos espectros Mössbauer das amostras naturais, após o tratamento com DCB e aquecidas a 800,1000 e $1200{ }^{\circ} \mathrm{C}$, medidos à temperatura ambiente. Área 1 e Área $2(\%)$ área sub-espectral relativa; $\mathrm{H}_{\mathrm{hfl}}$ e $\mathrm{H}_{\mathrm{hf2}}(\mathrm{kOe})=$ Campo magnético hiperfino; $\mathrm{E}_{\mathrm{Q}}(\mathrm{mm} / \mathrm{s})=$ Desdobramento quadrupolar; $\mathrm{IS}(\mathrm{mm} / \mathrm{s})=$ Desvio isomérico.

[Table II - Hyperfine parameters derived from the RT spectra of the natural DV sample before and after extractable Fe and burned at 800,1000 and $1200{ }^{\circ} \mathrm{C} . H_{h f}=$ hyperfine field; $2_{Q}=$ quadrupole shift; IS = isomer shift relative to $-\mathrm{Fe}(\mathrm{mm} / \mathrm{s}), E_{Q}=$ quadrupole splitting, and $A=$ relative area.]

\begin{tabular}{ccccccccccc}
\hline Amostra & $\mathrm{H}_{\mathrm{hf1}}$ & Área1 & $\mathrm{H}_{\mathrm{hf2}}$ & Área2 & $\mathrm{E}_{\mathrm{Q}}$ & $\mathrm{IS}$ & Área & $\mathrm{E}_{\mathrm{Q}}$ & $\mathrm{IS}$ & Área \\
\hline DV1200 & 504 & 89 & - & - & 0,96 & - & 11 & - & - & - \\
DV1000 & 505 & 92 & - & - & 0,87 & - & 8 & - & - & - \\
DV800 & 507 & 100 & - & - & - & - & - & - & - & - \\
DV & 510 & 49 & 280 & 42 & 0,59 & 0,35 & 9 & - & - & - \\
DVT & 511 & 33 & - & - & 0,44 & 0,27 & 57 & 2,31 & 1,15 & 10 \\
\hline
\end{tabular}


a não completa remoção dos óxidos de ferro ou que o $\mathrm{Fe}^{3+}$ esteja relacionado a substituições nos sítios octaédricos ou tetraédricos da caulinita, o que é compatível com as condições de formação do depósito em estudo, considerando que os difratogramas de raios $\mathrm{X}$ obtidos entre 22 e $25^{\circ} 2 \theta$ (Fig. 2A) apresentam a formação de uma banda, ou seja, a ausência das reflexões (020), (1-10) e (11-1). Essa banda é típica de

Tabela III - Teores percentuais de ferro total $\left(\mathrm{Fe}_{\text {total }}\right)$, hematita $(\mathrm{H})$, goethita $(\mathrm{Go})$ e $\mathrm{Fe}^{2+}$ nas amostras originais e tratadas com DCB.

[Table III - Percentage of total $\mathrm{Fe}, \mathrm{Fe}^{2+}$, hematite (H), goethite (Go) in the original samples before and after extractable $\mathrm{Fe}$.]

\begin{tabular}{ccccc}
\hline Amostra & $\mathrm{Fe}_{\text {total }}$ & $\mathrm{H}$ & $\mathrm{Go}$ & $\mathrm{Fe}^{2+}$ \\
\hline $\mathrm{DV}$ & 22,5 & 16 & 18 & - \\
$\mathrm{DVT}$ & 0,61 & 0,3 & 0,6 & 0,06 \\
\hline
\end{tabular}
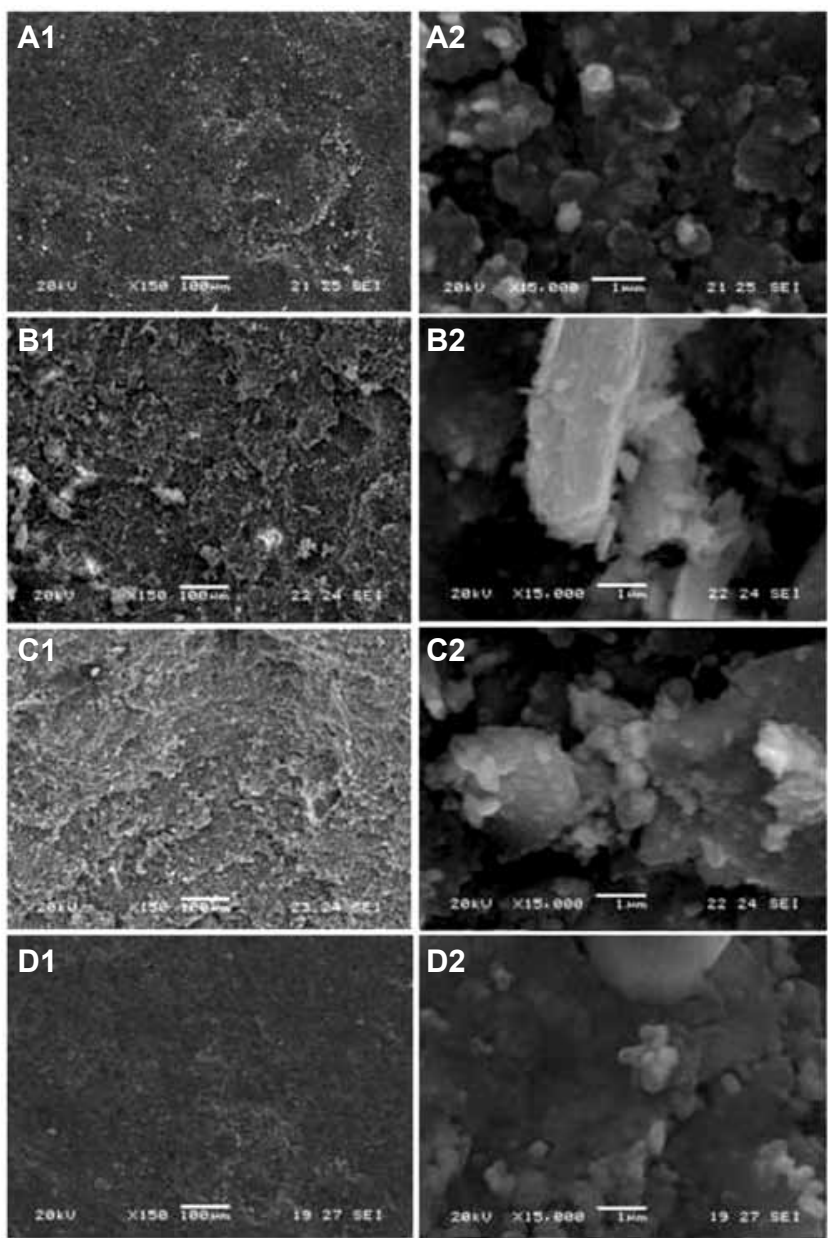

Figura 5: Fotomicrografias de microscopia eletrônica de varredura da amostra DV natural (A1 e A2) e queimada a 800 (B1 e B2), 1000 (C1 e C2) e $1200{ }^{\circ} \mathrm{C}(\mathrm{D} 1 \mathrm{e}$ D2). As fotomicrografias foram tomadas com aumento de 150 vezes para as amostras A1, B1, C1 e D1 e de 15000 vezes para as amostras A2, B3, C3 e D2.

[Figure 5: SEM micrograph of the raw materials (A1 and A2) and specimens burned at 800 (B1 and B2), 1000 (C1 and C2) e $1200{ }^{\circ} \mathrm{C}$ (D1 and D2).] caulinitas com defeitos estruturais, decorrentes da substituição de $\mathrm{Fe}^{3+}$ e $\mathrm{Fe}^{2+}$ em sua estrutura $[14,16]$.

Os parâmetros Mössbauer derivados dos ajustes computacionais dos espectros (Tabela II) permitiram calcular os teores de hematita, goethita e de $\mathrm{Fe}^{2+}$, na amostra natural com base nas áreas relativas de cada sub-espectro e dos teores de ferro total (Tabela III).

O aumento do tamanho do grão na amostra DV, que é uma das características da sinterização [17], pode ser observado nas microfotografias obtidas através do MEV (Fig. 5). À medida que a amostra passa pelo processo de sinterização, também se pode notar a presença de microfissuras nos corpos cerâmicos. A amostra prensada natural, sem tratamento térmico, se constitui principalmente de aglomerados de bordas irregulares e formas arredondadas, resultante do agrupamento de partículas de morfologia pseudo-hexagonal, e também apresenta formato laminar característico dos cristais de caulinita. Após sinterizada, a amostra apresenta em sua estrutura maior compactação e aglomeração das partículas, o que se torna mais visível quanto maior a temperatura de sinterização. Os tratamentos térmicos conservam a disposição laminar dos aglomerados dos cristais, entretanto em menor intensidade, pois as elevadas temperaturas a que a amostra foi submetida podem promover a delaminação estrutural das caulinitas.

O mapeamento composicional por EDS da amostra aquecida a $1200{ }^{\circ} \mathrm{C}$ apresenta os teores dos elementos $\mathrm{Fe}, \mathrm{Si}$ e $\mathrm{Al}$ distribuídos de forma homogênea na superfície do material relacionado a presença de mulita, e algumas concentrações de Fe relacionados a maiores cristais de hematita.

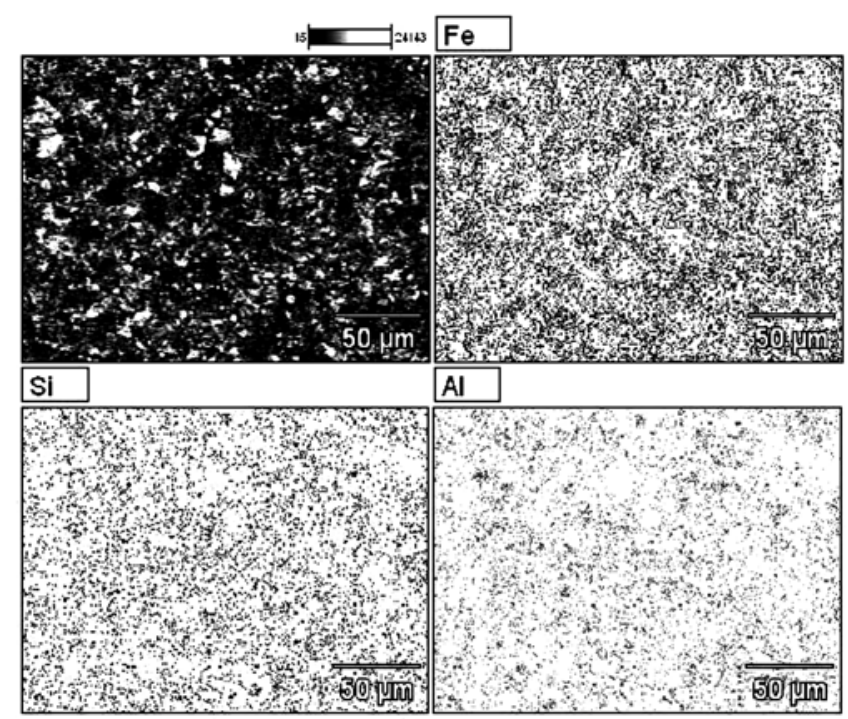

Figura 6: Distribuição dos elementos $\mathrm{Al}, \mathrm{Si}$, e Fe, obtidos por EDS, da amostra aquecida a $1200^{\circ} \mathrm{C}$.

[Figure 6: Distribution of chemical elements $\mathrm{Al}$, Si and Fe of the specimen burned at $1200^{\circ} \mathrm{C}$.]

\section{CONCLUSÕES}

A amostra DV é predominantemente constituída pelos óxidos $\mathrm{SiO}_{2}$ e $\mathrm{Al}_{2} \mathrm{O}_{3}$, seguido pelo elemento $\mathrm{Fe}$, presente na 
forma de óxidos e hidróxidos. A análise de espectroscopia Mössbauer, antes e após tratamento DCB, identifica a presença de $\mathrm{Fe}^{3+}$ dos compostos ferruginosos como hematita e goethita ou substituinte de sítios octaédricos da caulinita, e o $\mathrm{Fe}^{2+}$ como possível substituinte de sítios tetraédricos e octaédricos da caulinita, o que é compatível com as condições de formação do depósito. O processo de sinterização mostra uma desestruturação da caulinita, assim como também a transformação da goethita em hematita, cujo tamanho dos cristais crescem mais de 5 vezes. O teor elevado em Fe na amostra DV acarreta a formação da mulita formada da solução sólida de $\mathrm{Fe}_{2} \mathrm{O}_{3} \operatorname{com~} \mathrm{Al}_{2} \mathrm{O}_{3}$, o que resulta em produto resistente e de maior estabilidade térmica.

\section{AGRADECIMENTOS}

Os autores agradecem a contribuição financeira da FAPEMIG (Fundação de Amparo à Pesquisa do Estado de Minas Gerais) e do CNPq (Conselho Nacional de Desenvolvimento Científico e Tecnológico).

\section{REFERÊNCIAS}

[1] A. M. Morales-Carrera, M. G. Peralta-Sánchez, A. F. D. C. Varajão, M. M. Ferreira, "Aproveitamento de argilas cauliníticas naturais e com aditivos do Quadrilátero Ferrífero na obtenção de materiais cerâmicos", Eds.: E. B. Alvarado, M. J. Dominguez-Cuesta, "Técnicas Aplicadas a la Caracterización y Aprovechamiento de Recursos Geológico-Mineiros", vol. 1, CYTED e IGME, Madrid, 197 (2010) 160.

[2] M. G. Peralta-Sánchez, A. M. Morales-Carrera, A. F. D. C. Varajão, M. M. Ferreira, Viabilidade do uso de argilas cauliníticas do Quadrilátero Ferrífero para a indústria cerâmica, Cerâmica 57, 343 (2011) 254.

[3] M. M. Ferreira, Caracterização de argilas cauliníticas do Quadrilátero Ferrífero visando seu potencial de aplicação na indústria de cerâmica, Diss. de Mestrado, Departamento de Geologia - Escola de Minas- UFOP, Ouro Preto, Brasil (2010) 95.

[4] M. do C. Santos, Caracterização dos depósitos argilosos da porção centro-sul do Sinclinal Moeda, Quadrilátero Ferrifero, MG: macromorfologia, micromorfologia, cristaloquímica, gênese e considerações industriais, Tese Dr., Departamento de Geologia, Escola de Minas, UFOP,
Ouro Preto, MG (2003) 199.

[5] L. A. Gaspar Jr., Adição Experimental de Novos Materiais às argilas da Região do Pólo Cerâmico de Santa Gertrudes $(S P)$, Tese Dr., Departamento de Petrologia e Metalogenia, Instituto de Geociências e Ciências Exatas, Universidade Estadual Paulista, Campus de Rio Claro, SP (2003) 170.

[6] O. P. Mehra, M. L. Jackson, Iron oxide removal from soil and clays by a dithionite-citrate system buffered with sodium bicarbonate, Clays Clay Miner. 7 (1960) 317-327.

[7] G. P. Santana, J. D. Fabris, A. T. Goulart, D. P. Santana, Magnetite and its transformation to hematite in a soil derived from steatite, R. Bras. Ci. Solo 25, 1 (2001) 22.

[8] G. M. Da Costa, M. F. De Jesus Filho, X-ray differential line broadening on tabular haematites, J. Mater. Sci. 27 (1992) 6116.

[9] J. Morales, J. L. Tirado, M. Macias, Changes in Crystallite Size and Microstrains of Hematite Derived from the Thermal Decomposition of Synthetic Akaganeite, J. Solid State Chem. 53 (1984) 303.

[10] F. Watari, J. Van Landuyt, P. Delavignette, S. Amelinckx, N. Igata, X-ray Peak Broadening as a Result of Twin Formation in Some Oxides Derived by Dehydration, Phys. Stat. Sol. 73 (1982) 215.

[11] H. P. Klug, L. E. Alexander, "X-ray procedures for polycrystalline and amorphous materials", 2 $2^{\text {nd }}$ Ed., John Wiley Sons Inc., New York, EUA (1974).

[12] G. W. Brindley, G. Brown, "Cristal Structures of Clay Minerals and their X-ray Identification", Mineralogical Soc., London, Inglaterra (1980) 495.

[13] R. C. Mackenzie, "The differential thermal investigation of clays", Mineralogical Soc., London, Inglaterra (1957) 456.

[14] R. E. Grim, “Applied clay mineralogy”, McGraw-Hill Publ. Co. Ltd., New York, EUA (1962) 422.

[15] A. I. Avgustink, Cerámica Reverté S.A., Barcelona, Espanha (1983) 726.

[16] D. N. Hinckley, Variability in "cristallinity" values among the kaolin deposits of Georgia and South Carolina, Clays Clay Miner. 2 (1963) 229-235.

[17] L. Van Vlack, "Physical Ceramic for Engineers", Addison-Wesley Publ. Co., Inc. Trad.: "Propriedades dos Materiais Cerâmicos", Ed. Edgar Blücher Ltda., S. Paulo, SP (1973) 94.

(Rec. 17/12/2010, Rev. 28/03/2011, 23/05/2011, Ac. 24/05/2011) 